Die 7 Disziplinen im Sales-Management 
Nikolaus A. Rauch

\section{Die 7 Disziplinen im Sales-Management}

Eine Anleitung für nachhaltige Kundenund Geschäftsentwicklung im Vertrieb

Mit Zeichnungen von Jan Myszkowski

Springer Gabler 
Nikolaus A. Rauch

München

Deutschland

ISBN 978-3-658-04231-8

ISBN 978-3-658-04232-5 (eBook)

DOI 10.1007/978-3-658-04232-5

Die Deutsche Nationalbibliothek verzeichnet diese Publikation in der Deutschen Nationalbibliografie; detaillierte bibliografische Daten sind im Internet über http://dnb.d-nb.de abrufbar.

\section{Springer Gabler}

(C) Springer Fachmedien Wiesbaden 2016

Das Werk einschließlich aller seiner Teile ist urheberrechtlich geschützt. Jede Verwertung, die nicht ausdrücklich vom Urheberrechtsgesetz zugelassen ist, bedarf der vorherigen Zustimmung des Verlags. Das gilt insbesondere für Vervielfältigungen, Bearbeitungen, Übersetzungen, Mikroverfilmungen und die Einspeicherung und Verarbeitung in elektronischen Systemen.

Die Wiedergabe von Gebrauchsnamen, Handelsnamen, Warenbezeichnungen usw. in diesem Werk berechtigt auch ohne besondere Kennzeichnung nicht zu der Annahme, dass solche Namen im Sinne der Warenzeichenund Markenschutz-Gesetzgebung als frei zu betrachten wären und daher von jedermann benutzt werden dürften. Der Verlag, die Autoren und die Herausgeber gehen davon aus, dass die Angaben und Informationen in diesem Werk zum Zeitpunkt der Veröffentlichung vollständig und korrekt sind. Weder der Verlag noch die Autoren oder die Herausgeber übernehmen, ausdrücklich oder implizit, Gewähr für den Inhalt des Werkes, etwaige Fehler oder Äußerungen.

Lektorat: Manuela Eckstein

Zeichnungen: Jan Myszkowski

Gedruckt auf säurefreiem und chlorfrei gebleichtem Papier

Springer Fachmedien Wiesbaden ist Teil der Fachverlagsgruppe Springer Science+Business Media (www.springer.com) 
„Wer die Menschen behandelt, wie sie sind, macht sie schlechter. Wer sie aber behandelt, wie sie sein könnten, macht sie besser."

(Johann Wolfgang von Goethe) 


\section{Prolog}

„Ich weiß nicht mehr, wo es lang geht“, sagt mir der Geschäftsführer von Fang \& Stempel, einem Software-Unternehmen mit dreißig Mitarbeitern aus der Nähe von Hamburg. „Mein Kunde, seit zehn Jahren der Einzige, ist mit dem Fortschritt des neuen und wichtigsten Projekts unzufrieden." Durch einen Freund wurde ich gebeten, mich mit Herrn Fang zusammenzusetzen. Er ist kurz davor, alles hinzuschmeißen.

Meine erste Sicht auf das Projekt: Seit zwei Jahren wird bei Fang \& Stempel eine neue Steuerung für ein Massenprodukt im Medizinbereich entwickelt, aber es gibt keine Transparenz, weder inhaltlich noch beim Projektmanagement. „Gibt es ein Lastenheft?“ „Ja, seit eineinhalb Jahren.“ „Dann können wir doch daraus den Bearbeitungszustand ableiten, oder?“ „Na ja, die Geschäftsführer unseres Kunden kommen alle naselang mit neuen Anforderungen von Messen und Fachgesprächen.“ „Wer nimmt die auf beziehungsweise im Projekt ab?“ „Niemand! ... Na doch, unsere Entwickler.“ „Gibt es ein Angebot zur ursprünglichen Leistung, einen Auftrag?“ „Ja, ich habe dem Kunden eine Aufstellung der Komponenten und Phasen am Anfang geschickt“, sagt Geschäftsführer Fang, „Das war die Basis für den Auftrag.“ „Haben Sie nach dem Vertrag darüber nochmals gesprochen?“ „Nein, die Anforderung war doch klar!“” „Aber heute ist er unzufrieden?“ „Ja, daran ist er aber auch mit Schuld, er könnte uns ja klarer seine Abnahmekriterien nennen!“ „Und?“ „Dazu hat er keine Zeit ...“

So oder ähnlich entstehen Krisen in Unternehmen. Es fällt zunächst auf, dass das Hauptproblem nicht so sehr das Projektmissmanagement ist, sondern die ausschließliche Abhängigkeit von einem Kunden. Vor oder nach dem Vertragsabschluss: Ursache für solche Krisen ist fast immer ein mangelndes Kundenverständnis. Ja, nicht einmal auf die Idee zu kommen, den Kunden zu fragen, was er denn eigentlich will. Wo ist das ausgewogene Portfolio an Kunden und Projekten? Vielleicht kommt es erst gar nicht zu einem Projekt, dann fehlen die Aufträge. Fang hat es nicht geschafft, diesen einzigen Kunden mit seinem wichtigsten Auftrag abzuholen. Als erfahrener Experte könnte er die Krisenrisiken deutlich erkennen, bevor sie zu Krisenursachen werden. Und die liegen oftmals in der Beziehungsebene: Manager - Vertriebsmitarbeiter, Vertriebsmitarbeiter - Kunde, Nicht-Vertrieb - Vertrieb. 


\section{Sales Management aus der Krise}

Wenn Sie als Verantwortlicher für Vertrieb wie die Manager Johannes, Klaus, Robert und Głodny, die Sie später kennenlernen werden, glauben, dass sich wie bei Fang etwas in Ihrer Organisation ändern muss, haben Sie bereits einige der folgenden Aspekte in Unternehmungen entdeckt, die Grund für ausbleibenden Unternehmenserfolg sein könnten. Sehen Sie dazu, wie Sales Management Champions handeln: Die haben nämlich, im Zuge der hier dokumentierten Arbeit, folgende Voraussetzungen als Hebel für erstklassigen Vertrieb und damit für einen anhaltenden Erfolg in ihrem Unternehmen entdeckt:

1. Sie selbst, ihre Mitarbeiter oder auch ihre Führung haben die Veränderung des Käufermarktes nun richtig erkannt und ausreichend wahrgenommen. Sie sorgen nun dafür, dass es keine Zufriedenheit mehr mit dem bisher Erreichten gibt und somit durch Stolz auf die eigenen ,unübertrefflichen“ Leistungen eine Marketing-Kurzsichtigkeit, wie sie Theodore Levitt 1960 im heute noch gültigen Aufsatz „Marketing Myopia“ beschrieben hat (Levitt 2004), entstehen kann.

2. Sie haben erkannt, dass für das Unternehmen nie mehr die Anbieterperspektive mit Fokus auf die eigenen Produkte und Leistungen im Vordergrund stehen darf, sondern immer die Vorstellungen, Bedürfnisse und Visionen der Kunden, der Käufer ausschlaggebend sein müssen.

3. Sales Management Champions achten darauf, dass der Mangel an Bereitschaft oder gar die Unfähigkeit abgebaut wird, über Funktionsbereiche hinweg effizient zu kommunizieren. Vertrieb und Leistungserbringung haben dann kein Kommunikations- und Schnittstellenproblem mehr: Damit sind Produktion oder Dienstleistungsbereich ausreichend mit den Nachbarabteilungen vernetzt.

4. Sie sorgen - daraus resultierend - dafür, dass genügend Detailkenntnis der Leistungsmerkmale und Nutzenargumente im Vergleich zum Wettbewerb in der Zusammenarbeit fachübergreifend entsteht.

5. Es gehen keine vielversprechenden Projekte mehr in der zuvor dominierenden Angebotsmaschine des Proposal Managements verloren, da sie ausreichend selektiert haben, wo sie ein Angebot abgeben wollen und wo nicht - weil sie durch die Gratwanderung zwischen gerade mal ausreichender Kenntnis oder Berücksichtigung des Anbietermarktes und dem Druck in den Geschäftsdurchsprachen, eine positive Umsatzvorhersage zu liefern, das Gespür dafür wiedergewonnen haben, wo es sich wirklich lohnt.

6. Vertriebsorganisationen von Sales Management Champions haben gelernt, transaktionalen Verkauf abzulegen. Diese Methode war einst, mit dem Alleinstellungsmerkmal der einzige Anbieter dieser Leistung zu sein, absolut legitim: Es ging damals darum, Anfragen fehlerfrei zu beantworten. Für den relationalen Vertrieb, der sich auf den Kunden ausrichtet, der die Rahmenbedingungen und Menschen beim Kunden in seine Überlegungen einbezieht, wurde Ihnen die methodische Kenntnis nun vermittelt. Einen Kunden organisatorisch zu erschließen, ist heute gefragt, im Fokus stand früher dagegen immer das Produkt mit seinen Merkmalen, die sich messbar beschreiben ließen. Und das lief bisher immer gut: 50 auf 50 auf $50 \mathrm{~cm}, 70 \mathrm{~g}$ schwer und blau. Die Philosophie 
des Autobauers Ford, dass seine Kunden jedes Fahrzeug („Model T“) haben könnten, Hauptsache es sei schwarz, scheint tief eingebrannt in die Vertriebsseele. Erst als ihm General Motors und Chrysler mit hübscheren, leistungsfähigeren Modellen den Rang abliefen, konnte ihn sein Sohn, Edsel Ford, überzeugen, ein neues Auto zu bauen.

7. Sales Management Champions haben erkannt, dass sich mittel- und langfristig etwas ändern muss, weil der Kunde Ansprüche stellt, die sie mit der bisherigen Vorgehensweise nicht befriedigen konnten. Und so können sie Mitarbeiter und Kunden zu Geschäften motivieren, weil sie diese neue Perspektive als Triebfeder für Engagement bereitstellen.

Wie viele Führungskräfte im Vertrieb haben Sales Management Champions sich oft zunächst einmal darin bestätigt gesehen, dass ihre Fähigkeiten als Topverkäufer, ihre Kunst des Fire Fightings, das kurzfristige Erreichen der Ziellinie garantieren - doch für einen langfristigen Vertriebserfolg müssen sie heute eher die Rolle eines Architekten übernehmen. Sie wurden wegen ihrer vertrieblichen Expertise und Leistungen in die Leitungsrolle geholt, ohne dass ihnen irgendjemand erklärt hätte, dass im Vertriebsmanagement andere Regeln herrschen als beim Kunden vor Ort. Das haben sie eigenständig nachgeholt und sich als Entscheider im Vertrieb, im Vertriebsmanagement ausbilden lassen. Sie haben neben den üblichen Führungstrainings nach Hilfestellung für ihre Rolle als Coach gesucht - einem Instrument, um die Verkaufssicht der Mitarbeiter zu schärfen und gegebenenfalls zu verändern (Richardson 2008, S. 16).

In einer solchen Weiterbildung wurde den Sales Management Champions das Zusammenspiel von Management und Leadership-Fähigkeiten für die Praxis vermittelt. Nun fühlen sie sich verantwortlich für die aktive Weiterentwicklung der Vertriebsmitarbeiter und das Team Building zwischen den Einheiten und sehen darin einen Vorteil für Wachstum und Prosperität. Es gibt keinen Zweifel mehr daran, dass das Vertriebsmanagement weit über die Administration von Zahlen, Daten, Fakten sowie die messbare Steuerung einer Mannschaft hinausgeht. Sales Management Champions erhöhen durch konstruktive Fragen die Erfolgswahrscheinlichkeit von Geschäftsoptionen; sie haben die Rolle des Vertriebscoachs als wichtiges Hilfsmittel für sich erkannt. Bei einem erwachsenen Menschen Kritik an dessen intellektueller Leistung zu üben, trauen sie sich heute zu. Für sie ist es keineswegs mehr peinlich, wenn Sie Kundentermine oder Verhandlungen beobachten, analysieren und kritisieren, weil dieses „Coaching“ - absolut diskret und professionell die Glaubenssätze, d. h. die individuellen Theorien, warum etwas so und nicht anders ist, der Mitarbeitern und des Unternehmens berücksichtigt.

Während Sie dies lesen, bezweifeln Sie, dass die sogenannten Sales Management Champions, aufgrund der umfangreichen administrativen Verantwortlichkeiten, an denen auch Sie, lieber Leser, gemessen werden, Zeit für derartigen philosophisch-psychologischen Schnickschnack haben. Oder Sie glauben, dass Sie den Vertrieb „nur“ mit Eigenschaften wie Abstraktion, Menschennähe und Konsequenz erfolgreich führen können. Aber blass ist alle Theorie. Alles in allem erkennen Sie nun, wie viele Werkzeuge Sie benötigen, um die bisher aufgeführten Schwächen, Fragestellungen und Hindernisse zu 
bearbeiten, in der Sache und im Prozess, um mit Coaching Vertriebsentwicklung erfolgreich zu gestalten. Zweifellos gibt es immer mehrere erfolgreiche Philosophien, um Vertrieb zu gestalten. Doch es bleibt dabei, dass der Wurm dem Fisch und nicht dem Angler schmecken muss. Berücksichtigen Sie aber bitte, dass es verschiedene Fische, Gewässer und Angelruten gibt.

Der Wandel zu ausdrücklicher Kundenorientierung, weg von Kontakt und Abschluss hin zu inhaltlich anspruchsvollen partnerschaftlichen Beziehungen, betrifft viele auch der selbstbewusst auftretenden Speaker und Trainer. Sales Management findet heute in vielen Unternehmen nur rudimentär statt. Wer erkannt hat, dass professionelle Vertriebsführung das Wachstum und den Ertrag ohne wachsende Ausgaben überdurchschnittlich steigert, wird aus diesem Buch einige Anregungen ziehen und sich nachhaltig um sein Unternehmen verdient machen.

\section{Grundidee}

Die Grundzüge modernen Vertriebsmanagements und seiner Strategie beinhalten eine Reihe von positionellen Merkmalen, die - richtig aufgefasst und eingeschätzt - eine passende Bewertung jeder aktuellen vertrieblichen Lage erlauben und somit die nötigen Voraussetzungen dafür schaffen, den objektiv besten Plan zu finden. Diese positionellen Merkmale lassen sich in drei Gruppen einteilen:

- schwache und starke Werteargumente

- Vertriebsstruktur

- Wirkung und Flexibilität der Mitarbeiter

Bedenken Sie dabei, dass diese positionellen Merkmale wechselseitig voneinander abhängen und als System betrachtet werden müssen. Vor Ihnen liegt ein Vertriebslese- und -lernbuch, das Sie bei der Bewertung dieser positionellen Merkmale und der Auswahl der daraus resultierenden geeigneten Vorgehensweisen begleiten wird. Wie im richtigen Leben werden Sie als Geschäftsführer, Vertriebsleiter, Unternehmer in das vertriebliche Geschehen hineingeworfen und sollen sich durch das Netz an bereitgestellten Informationen und Geschichten einen eigenen Weg erarbeiten.

\section{Mitwirkende und ihre Unternehmen}

Vier Vertriebsverantwortliche: ein Vertriebsmanager, ein Bereichsleiter, ein Geschäftsführer und ein Vorstand. Darüber hinaus zwei Angestellte und zwei Unternehmer aus den Bereichen Hightech, Beratung, Mittelstand und Industrie. Sie alle werden in ihrer aktuellen Situation vorgestellt. Und alle befinden sich in kritischen Management- und Unternehmenssituationen:

Dr. Johannes Mannsheim, Bereichsleiter bei der Steam Success International KG (s. Kap. 7.3.1, s. 330), mit seinen Mitarbeitern: 
- dem marktnahen Antoine aus Lille in Frankreich (s. Kap. 1.3.2, S. 14)

- dem kontaktfreudigen Erik aus Norköpping in Schweden (s. Kap. 2.3.1, S. 72)

- dem überzeugenden Guido aus Köln in Deutschland (s. Kap. 4.3.3, S. 197)

- dem kundenfokussierten Marcos aus Spanien (s. Kap. 6.3.1, S. 274)

- dem anpackenden Mikkel aus Kalundnorg in Dänemark (s. Kap. 5.3.2, S. 229)

- dem technisch versierten Thomé aus Basel in der Schweiz (s. Kap. 3.3.1, S. 138)

Klaus de Yong, Geschäftsführer bei der Fournier Système GmbH (s. Kap. 7.3.2, S. 333), mit seinen Vertriebsmitarbeitern:

- dem rastlosen Bailey aus Redding in Großbritannien (s. Kap. 2.3.2, S. 75)

- der hochkommunikativen Elène aus Huizingen in Belgien (s. Kap. 3.3.3, S. 145)

- dem einfühlsamen Günter aus Alzenau in Deutschland (s. Kap. 6.3.4, S. 285)

- dem materialistischen Owen aus Manchester in Großbritannien (s. Kap. 5.3.3, S. 232)

- dem angriffslustigen Steve aus Monterey in Kalifornien in den USA (s. Kap. 4.3.2, S. 194)

- dem entschlossenen Tobiáš aus Prag in Tschechien (s. Kap. 1.3.4, S. 20)

Głodny Wilk, Vorstand und Inhaber der PIP Power Inside Production S. A. (s. Kap. 7.3.3, S. 335), mit seinen Mitarbeitern:

- der unternehmerischen Agnieska aus Gdańsk in Polen (s. Kap. 1.3.3, S. 17)

- dem optimistischen Dariusz aus Wrocław in Polen (s. Kap. 3.3.4, S. 274)

- der leidenschaftlichen Janina aus Moskau in Russland (s. Kap. 2.3.3, S. 148)

- dem kooperativen Marcin aus Bolesławiec in Polen (s. Kap. 6.3.3, S. 282)

- dem strukturierten Roman aus Gdańsk in Polen (s. Kap. 4.3.3, S. 201)

- der beharrlichen Zuzanna aus Bratislava in der Slowakei (s. Kap. 5.3.1, S. 225)

Robert Ganges, geschäftsführender Partner bei der Terra Consult GmbH (s. Kap. 7.3.4, S. 338), mit seinen Partnern:

- der ausgefuchsten Alicia Christiana aus Dedham in Massachusetts in den USA (s. Kap. 6.3.2, S. 278)

- der optimistischen Doreen aus Dublin in Irland (s. Kap. 4.3.1, S. 191)

- dem weitblickenden Frank aus Halle in Deutschland (s. Kap. 1.3.1, S. 10)

- dem eigennützigen Noé aus Marseille in Frankreich (s. Kap. 5.3.4, S. 236)

- dem verständnisvollen Stefano aus Padua in Italien (s. Kap. 2.3.4, S. 82)

- dem begeisterten Uwe aus Münster in Deutschland (s. Kap. 3.3.2, S. 141)

Anhand der folgenden Fallbeispiele soll veranschaulicht werden, wie diese Personen die jeweiligen, durch das Management vorgegebenen Prinzipien vertrieblichen Handelns umsetzen. In Interviews beschreiben die Chefs jeweils sechs typische Vertriebsmitarbeiterprofile. Wie in der Realität auch, wechselt sich die Arbeit am Markt und mit den Mit- 
arbeitern ab. Sollten Sie Ähnlichkeiten mit real existierenden Firmen, Personen oder Situationen aus Ihrem Umfeld entdecken, ist das durchaus beabsichtigt, aber dennoch rein zufällig. Dann hätte sich die Arbeit an diesem Buch gelohnt.

\section{Steam Success International KG: Dr. Johannes Mannsheim, Bereichsleiter}

Heute wird sich die Stadt Basel entscheiden. Dr. Johannes Mannsheim wartet auf den Statusbericht des Schweizer Account Teams. Mit dem Auftrag von über 20 Mio. Schweizer Franken hätte er seinen Plan in diesem Markt übererfüllt und könnte Belgien kompensieren, wo nach drei Projektverlusten zum wiederholten Male der Forecast nach unten korrigiert wurde.

Steam Success International ist voll in den Strudel des Verdrängungswettbewerbs geraten: Es gewinnt immer der günstigste Anbieter, das ist mittlerweile in allen Ländern so, auch im bisherigen „Schlaraffenland“ Österreich. Allerdings kann er sich auf den dortigen Vertriebsleiter verlassen, der mit mehr als 50\% Marktanteil und einem respektablen Rohertrag von über $35 \%$ seinen Markt im Griff hat.

Da kommt der Anruf: Leider wurde das Projekt in der Schweiz an den größten Wettbewerber vergeben. Ratlosigkeit macht sich im Gesicht von Johannes breit. Was erzählt er nun nächsten Montag in der Telefonkonferenz dem Geschäftsführer?

Es ist Mittwoch 16.00 Uhr, in einer Viertelstunde muss er zum Frankfurter Flughafen, dort trifft er den Leiter der Entwicklung des Anlagenbauers Helsingoer. Mit diesem Geschäft könnte er die Umsatzschwäche der Dänen ausgleichen. Er hat gelernt: Wenn er nicht selbst anpackt, erreicht er seine Zahlen nicht.

Ach ja, eine Verlustanalyse in der Schweiz wäre hilfreich, aber jetzt geht es nach Frankfurt und von dort nach Dublin. Der Hilfeschrei der dortigen Leitung zwingt zum Handeln: Er muss selbst hin und Hand anlegen, schließlich ist er nicht umsonst als Top Scorer in der Firma für diesen Sales Management Job ausgewählt worden.

\section{Fournier Système GmbH: Klaus de Yong, Geschäftsführer}

Klaus de Yong steht mächtig unter Druck: Bereits im dritten Jahr liefert er mit seinen Druckern kein Ergebnis mehr ab. Am Markt herrscht schon seit langer Zeit reiner Preiskampf in allen Produktkategorien. Großkundenprojekte werden nicht gewonnen, können nicht abgewickelt werden oder gehen verloren. Der dramatische Margenverfall im Produktgeschäft ist bereits da oder zeichnet sich allerorten ab.

Die chinesische Mutter des Unternehmens, Chin Hwan aus Daxing, die den französischen Hersteller vor fünf Jahren gekauft hat, erwartet von de Yong höhere Absatzzahlen, denn die Produktion in Daxing darf nicht stillstehen. Und die Wettbewerber wie HP, Xerox und andere schlafen nicht, im Gegenteil: Der Wettbewerb hat die Konvergenz von Druckern und Informationstechnologie zur Chefsache gemacht. Kooperationen und Kauf von Systemhäusern sind an der Tagesordnung.

Martin Powerbeck, Mitglied der Geschäftsführung und Marketingchef bei Fournier, weiß, dass sich die Branche im Umbruch befindet und Printing vor einem Paradigmenwechsel steht: Wer die Konvergenz hin zu IT/ITK-Consulting nicht mitmacht, den bestraft 
der Markt. Er hat mit den Managed Print Services ein Rundum-sorglos-Paket für den Mittelstand entwickelt - technisch brillant und am Markt erwünscht. Die Account Manager und Print Consultants, wie Klaus de Yong seine Verkäufer nennt, sollen diese Leistungen in den Markt tragen. Strategischer Vertrieb soll für nachhaltige Kundenbeziehungen sorgen und wegführen vom Tropf der Distributoren und Systemhäuser. Doch genau daran beißt sich das Management die Zähne aus. Wie verkauft man statt Produkten Services, wie lernt eine Organisation von exzellenten Transaktionsverkäufern, Relationsvertrieb zu machen? Denn wie Powerbeck sagt: „Service verkauft sich nicht im Regal wie eine Waschmaschine." Zudem hat Fournier durch die Integrationen von kleinen Systemhäusern mit organisatorischer Instabilität zu kämpfen.

Powerbecks Paketlösung wird leider nur halbherzig am Markt angeboten, und damit entsteht für Chin Hwan auch kein Rücklauf der Investitionen. Es wächst die Kluft zwischen der Geschäftsprognose der Holding und de Yongs Geschäftserwartung aus dem Midterm-Plan hinsichtlich Umsatz und Ertrag. Die vorhandene Consulting-Kapazität für das Lösungsgeschäft wird nicht wirklich abgefragt, und damit entsteht die Gefahr, dass Fournier gute Leute verliert. Das Marketingkonzept verkümmert, Widerstand regt sich in der Verkaufsorganisation, Stückzahlen bei steigenden Renditevorstellungen zu erhöhen ist unmöglich. Der Posten des Vertriebsmanagers ist vakant.

\section{PIP Power Inside Production S. A.: Glodny Wilk, Vorstand und Inhaber}

Der Breslauer Unternehmer Głodny Wilk steht mit PIP Power Inside Production am Scheideweg. Die EU hat sein Unternehmen bisher stark gefördert, heute ist er Arbeitgeber für zweihundertfünfzig Mitarbeiter in Wrocław und Sponsor des Handballnationalteams. Seine Geräteproduktion begann Głodny mit seiner Frau Basia in der heimischen Küche in Katowice, heute hat er ein nach allen Regeln des Qualitätsmanagements vorbildlich entwickeltes Produktionsverfahren.

Doch die Wachstumsraten sind eingebrochen, das Ergebnis flacht ab, er hängt vom Mittelstand ab - und der ist, außer in Deutschland, im Keller. Seine Vertriebsorganisation ist vornehmlich auf den indirekten Kanal ausgerichtet, ein sogenannter Account Manager verkauft vereinzelt Lösungen an Handelsketten oder Großunternehmen. Er merkt, der Hebel in den Markt ist zu kurz: Wenn die Endkunden Halsschmerzen haben, bekommen die Distributoren und Systemhäuser Schnupfen und PIP eine Lungenentzündung. Marktschwäche und eine fehlende Wachstumsstrategie, Wilk weiß genau, dass nur ein radikaler Umbau PIP retten kann.

PIP befindet sich in einem immer härter werdenden Verdrängungswettbewerb. Anzeichen dafür waren bereits vor sechs Monaten zu erkennen. Die aktuelle Vertriebskompetenz der fünfundzwanzig Innendienstler des Unternehmens kann diese Entwicklung nicht abfedern. Momentan fängt das Distributionsgeschäft den zunehmenden Umsatz- und Ertragsrückgang im Vertrieb der Eigenmarke „Moc dwójka“ nicht auf. Die Konsolidierungswelle im Distributionsmarkt wird aber über kurz oder lang auch die PIP S. A. erreichen. Fehlende Neukundengewinnung und Account-Entwicklung haben erst zum Umsatzstillstand, dann zum Umsatzrückgang geführt. Der Führungsstil des Geschäftsführers 
hat keine Kultur des selbstständigen Handelns zugelassen, dadurch kommen nur wenige Impulse aus der Mannschaft. Im Vorstandsteam diskutieren wir gemeinsam mögliche Lösungen, allerdings beherrscht die Performance des nationalen Handballteams $30 \%$ des Gesprächs.

\section{Terra Consult GmbH: Robert Ganges, Geschäftsführer und Teilhaber}

Robert Ganges sitzt erschöpft in seinem Maserati Quattroporte. Es ist Freitag, der 30. Oktober, die monatliche Durchsprache der Projekte mit den Partnern hat wie so oft eineinhalb Stunden länger gedauert als geplant. Wieder verlässt er das Büro mit dem hilflosen Gefühl, der Steuerung seiner Partner nicht wirklich gewachsen zu sein. Glücklicherweise hat er noch einen Termin bei Blue Invest, wo er sich einen Auftrag abholen kann.

Aber der Reihe nach: Robert hat Terra Consult vor sechs Jahren gegründet, sein Dienstleistungsangebot hatte wie eine Bombe im Markt eingeschlagen. Nun hat er nach und nach zehn Partner an Bord geholt, die wie er den Markt aufrollen sollen. Doch das lief bisher ziemlich zäh. ,Der Markt ist doch da, reifer geht es nicht“, wird er in jeder der „Einzelbestrahlungen“, wie die Partner die monatlichen Durchsprachen nennen, nicht müde zu wiederholen. Da ist Noé, eigentlich pflegeleicht: Er erreicht seine Umsätze immer, aber mit hohem Aufwand. Da ist Frank, der Minimalist: Man scheint ihn immer zum Kunden tragen zu müssen, allerdings ist seine Ausbeute erstaunlich, mit der Trefferquote von über $30 \%$ ist er im Unternehmen zweifellos Benchmark. Da ist die instabile Doreen: Diesen Monat hat sie nicht einen Abschluss getätigt, und ihre Projektliste hat „Schwindsucht“, wie Robert kommentiert. Er selbst präsentiert zum Abschluss des Partnertages seine Zahlen: Fünfzig Anfragen, den geplanten Umsatz wieder um die Hälfte übererfüllt, seine Besuchsliste macht dem Dax alle Ehre. Mit zwanzig Top-CEO-Terminen ist er mit weitem Abstand der fleißigste Vertriebsmitarbeiter. Aber dann sind da noch die Unternehmenszahlen: Die geplanten Wachstumsraten sind wieder nicht erfüllt, doch darauf beruhte der Investitionsplan. Robert wird heute wieder das Abendessen mit Helga und den Kindern verpassen, dafür schließt er bei Dr. Heidegger von Blue Invest diesen Auftrag ab.

Wie Robert Ganges ergeht es vielen Start-up-Unternehmen: eine geniale Idee, eine außergewöhnliche vertriebliche Begabung und Fleiß. Das reicht gegebenenfalls für einen kurz- oder mittelfristigen Erfolg. Spätestens wenn das Wachstum kein Selbstläufer mehr ist, weil der erste Hype abflacht oder sich Wettbewerb einstellt, braucht es Managementintelligenz, denn die negativen Auswirkungen lassen nicht lange auf sich warten: Cashflow-Schwäche und Umsatzunvorhersehbarkeit. 


\section{Danksagung}

Ich möchte mich bei vielen Gesprächspartnern bedanken, angefangen bei Eckard, meinem väterlichen Freund, der mir viele entscheidende Hinweise gegeben hat, für die Gespräche mit Johannes (viel Vergnügen im Ruhestand!), Jürgen, Marcus und Rainer, die bei diversen Anwendungen, wenn man so will, Pate gestanden haben, bis zu einigen ,Versuchskaninchen“, die kritisch Probe lesen „durften“, wie Joachim, Erich, Wolfgang und andere mehr. Bei meinem Geschäftspartner und Freund Guido, der mir half, aus mancher Theorie Praxis zu machen und mit dem ich die Managementphilosophie teile, dass Unvermögen der Mitarbeiter in erster Linie eine Führungssache ist.

Auch wenn der eine oder andere Artikel in der Toskana unter Pinien mit Blick auf das Mittelmeer entstand, war es harte Arbeit, ein Studium, das mich nach fünfundzwanzig Praxisjahren demütig gemacht hat vor der Bandbreite vertrieblichen Handelns. Was meinen langjährigen Freund Jan angeht, so sprechen seine ideenreichen Bilder und Karikaturen in diesem Buch für sich.

Mein Dank gilt zu guter Letzt sechs Personen, ohne die das alles nicht gelungen wäre. Zum einen Michael Schickerling, meinem geduldigen Lektor, der nur selten darauf hinwies, dass er mir ja den hohen Aufwand prophezeit hätte, Manuela Eckstein, die mir immer wieder Vertrauen geschenkt hat, Stephanie, die meine Texte (40 Zeilen à 20 Wörter) entziffert, erfasst und in Form gebracht hat, meinem Sohn Johannes, der mein Chaos an Literatur ,auf die Reihe brachte“ sowie, ganz wichtig, Vati (Jahrgang 1921), dem ich viele Nächte der Reflexion verdanke und der die Veröffentlichung des Buchs leider nicht mehr erleben kann, und meiner lieben Frau Oda, die sehr viel ertragen musste. 


\section{Inhaltsverzeichnis}

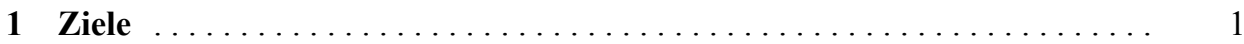

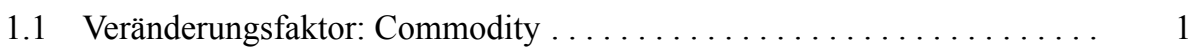

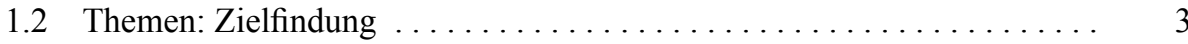

1.2.1 Strategieprozess $\ldots \ldots \ldots \ldots \ldots \ldots \ldots \ldots \ldots \ldots \ldots \ldots, 3$

1.2.2 Mission und Selbstverständnis: Wofür gibt es den Vertrieb? ..... 4

1.2.3 Vision und Ziele: Wohin wollen Sie vertrieblich? ........... 5

1.2.4 Werte als vertriebliches Zuhause: Wie arbeiten

Sie mit anderen zusammen? ................... 5

1.3 Profile: Stratege, Brancheninsider, Unternehmer und Manager ....... 10

1.3.1 Der Stratege . . . . . . . . . . . . . . . . . . . . . . 10

1.3.2 Antoine, der Brancheninsider . . . . . . . . . . . . . . . . . 14

1.3.3 Agnieska, die Unternehmerin . . . . . . . . . . . . . . . . . . 17

1.3.4 Tobiáš, der Manager ............................ 20

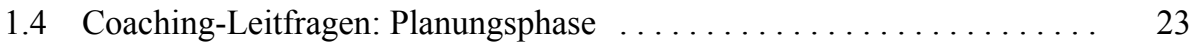

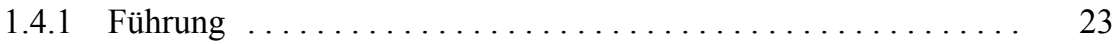

1.4.2 Mitarbeiter ............................... 24

1.4.3 Allgemeine, persönliche Fragen . . . . . . . . . . . . . . . 24

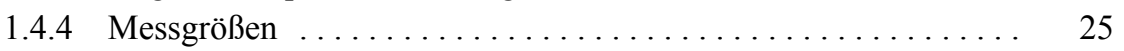

1.4.5 Leistungskennzahl: Market-Growth-Rate $\ldots \ldots \ldots \ldots \ldots \ldots .25$

1.5 Leadership: Vertriebsführung im Strategieprozess $\ldots \ldots \ldots \ldots \ldots .26$

1.5.1 Modelle: Bilder und Buchstaben zur Struktur . . . . . . . . . 26

1.5.2 Aus dem Sales-Management-Werkzeugkasten .......... 31

1.6 Anwendung und Ergebnisse: Strategieprozess $\ldots \ldots \ldots \ldots \ldots \ldots . . \ldots \ldots$

Literatur .................................... 35

2 Painpoints, zwingende Ereignisse und Projekte ................ 37

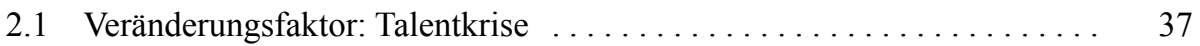

2.2 Themen: Ableitungen aus dem Leitbild für die Vertriebsarbeit ....... 39

2.2.1 Vertriebsziele und Vertriebsstrategie $\ldots \ldots \ldots \ldots \ldots \ldots \ldots . . \ldots \ldots$

2.2.2 Vertriebstaktik ............................ 43 
2.2.3 Vertriebsprozess, Pipeline und Vertriebstrichter . . . . . . . 45

2.2.4 Vertriebliche Priorisierung: Kundenstrukturanalyse ........ 52

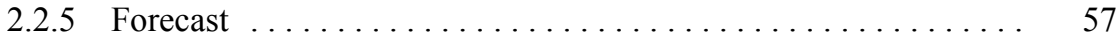

2.2.6 Kaufmotiv: Painpoints und Vision . . . . . . . . . . . . . 59

2.2.7 Kontaktaufnahme und Kaltakquise . . . . . . . . . . . . 63

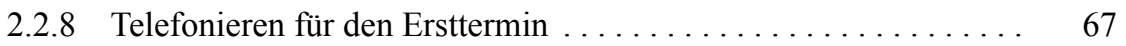

2.2.9 Sprache und Kommunikation $\ldots \ldots \ldots \ldots \ldots \ldots \ldots \ldots . \ldots \ldots$

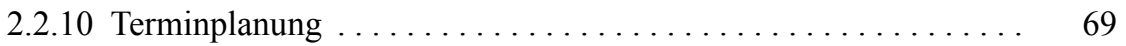

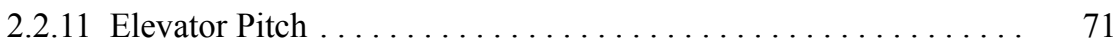

2.3 Profile: Versteher, Schrotflinte, Trommler und Freund . . . . . . . . . . 73

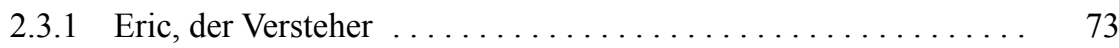

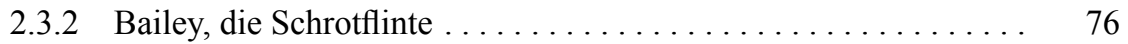

2.3.3 Janina, die Trommlerin $\ldots \ldots \ldots \ldots \ldots \ldots \ldots \ldots \ldots \ldots . \ldots \ldots$

2.3.4 Stefano, der Freund $\ldots \ldots \ldots \ldots \ldots \ldots \ldots \ldots \ldots \ldots \ldots . \ldots \ldots$

2.4 Coaching-Leitfragen: Kundenbearbeitung $\ldots \ldots \ldots \ldots \ldots \ldots \ldots$

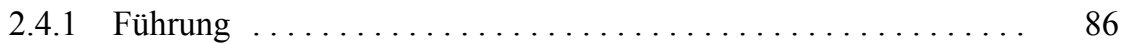

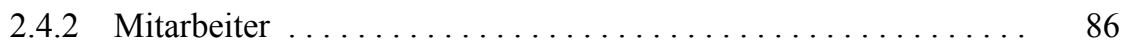

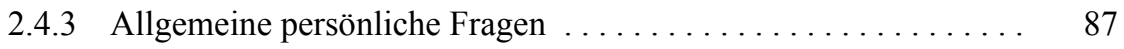

2.4 .4 Messgrößen ... . . . . . . . . . . . . . . . . . . . . . . 87

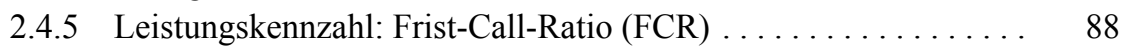

2.5 Leadership: Vertriebsführung für die Kundenarbeit $\ldots \ldots \ldots \ldots \ldots \ldots 8$

2.5.1 Stallgeruch: Netzwerke aufbauen ............... 88

2.5.2 Aus dem Sales-Management-Werkzeugkasten ........... 91

2.6 Anwendung und Ergebnisse: Marktbearbeitung . . . . . . . . . . 96

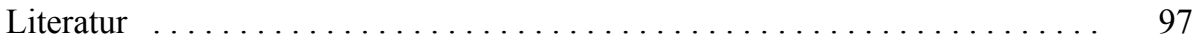

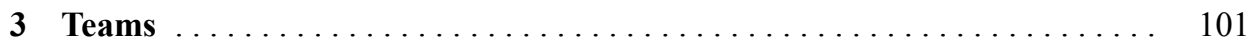

3.1 Veränderungsfaktor: Marktgeschwindigkeit $\ldots \ldots \ldots \ldots \ldots \ldots \ldots \ldots . \ldots 10 \ldots$

3.2 Themen: Persönlichkeit Rollen und Profile . . . . . . . . . . . . 103

3.2.1 Visitenkarte und andere identitätsstiftende Dinge . . . . . . . . 103

3.2.2 Leistungspotenzialeisberg: Monitoring und Assessment . . . . . . . 105

3.2.3 Entwicklung der Vertriebsmitarbeiter . . . . . . . . . . . . 106

3.2.4 Bilder, Geschichten und andere Erfahrungen . . . . . . . . . . . . 109

3.2 .5 NLP und Vergleichbares . . . . . . . . . . . . . . . . . . . . . . 112

3.2 .6 Zuhören .............................. 119

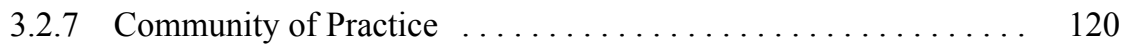

3.2 .8 Widerstände . . . . . . . . . . . . . . . . . . . . . . . . . 123

3.2 .9 Persönlichkeiten . . . . . . . . . . . . . . . . . . . . . . 124

3.2.10 Profil von Verkäufern und Account Managern . . . . . . . . . . 130

3.2 .11 Erkennen der Stärken . . . . . . . . . . . . . . . . . . 136

3.3 Profile: Techniker, Enthusiast, Kommunikator und Frohnatur . . . . . . . . 140

3.3.1 Thomé, der Techniker . . . . . . . . . . . . . . . 140

3.3.2 Uwe, der Enthusiast . . . . . . . . . . . . . . . . 143 
3.3.3 Elène, die Kommunikatorin . . . . . . . . . . . . . . . . . . . . . . . 147

3.3 .4 Dariusz, die Frohnatur . . . . . . . . . . . . . . . . . . 150

3.4 Coaching-Leitfragen: Arbeit mit den Vertriebsteams . . . . . . . . . . . . 154

3.4 .1 Führung ............................. 154

3.4 .2 Mitarbeiter . . . . . . . . . . . . . . . . . . . . . . . 154

3.4.3 Allgemeine, persönliche Fragen . . . . . . . . . . . . . . 155

3.4 .4 Messgrößen . . . . . . . . . . . . . . . . . . . 155

3.4.5 Leistungskennzahl: Human Capital Value Added (HCVA) . . . . . 155

3.5 Leadership: Reflexionen zur Arbeit mit den Vertriebsteams . . . . . . . . 156

3.5.1 Einflussfaktoren zur Sales Champions League . . . . . . . . . . . 156

3.5.2 Aus dem Sales-Management-Werkzeugkasten . . . . . . . . . . 157

3.6 Anwendung und Ergebnisse: Arbeitsweisen . . . . . . . . . . . . . 160

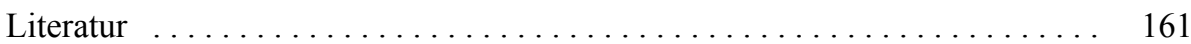

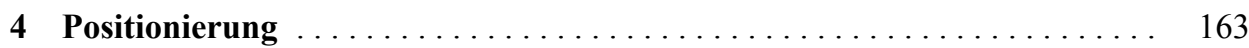

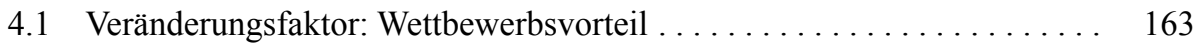

4.2 Themen: Umsetzen der Vertriebszyklen $\ldots \ldots \ldots \ldots \ldots \ldots \ldots \ldots \ldots . \ldots \ldots$

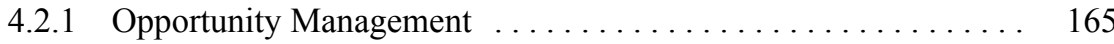

4.2 .2 Sales-Modelle ... . . . . . . . . . . . . . . . . . . . . . 166

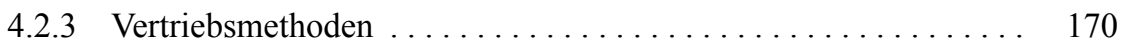

4.2 .4 Opportunity Check . . . . . . . . . . . . . . . . . . 182

4.2.5 Wettbewerbsvorteile und Geschäftsnutzen . . . . . . . . . . . . . 184

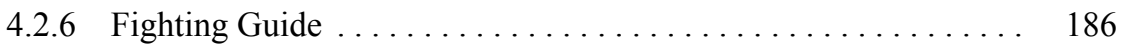

4.2.7 Unternehmerische Handlungslogik . . . . . . . . . . . . . 186

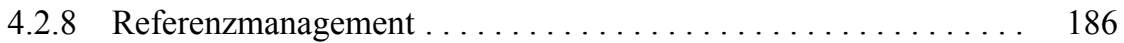

4.2 .9 Werte und Werteverfall ... . . . . . . . . . . . . . . . . . 190

4.3 Profile: Idealist, Wettkämpfer, Überredungskünstler und Ordentlicher . . 192

4.3.1 Doreen, die Idealistin . . . . . . . . . . . . . . . . . . . . . . . . 192

4.3.2 Steve, der Wettkämpfer . . . . . . . . . . . . . . . . . 195

4.3.3 Guido, der Überredungskünstler . . . . . . . . . . . . . . . . . . . . . 198

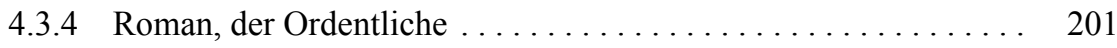

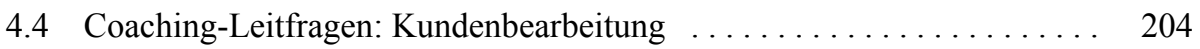

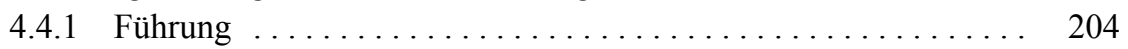

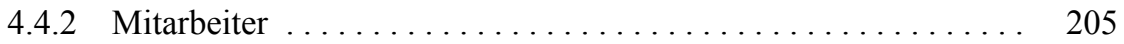

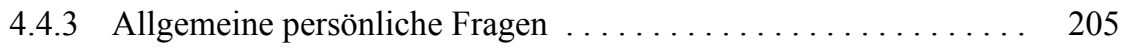

4.4 .4 Messgrößen .......................... 205

4.4.5 Leistungskennzahl: Net Promoter Score ${ }^{\circledR} \ldots \ldots \ldots \ldots \ldots \ldots . \ldots 206$

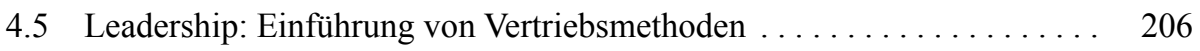

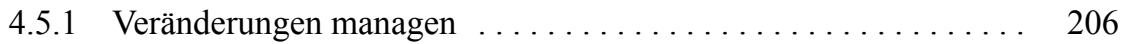

4.5.2 Aus dem Sales-Management-Werkzeugkasten . . . . . . . . . . 208

4.6 Anwendung und Ergebnisse: Methoden und Konzepte . . . . . . . . . . . 211

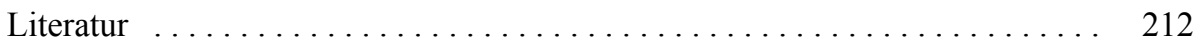




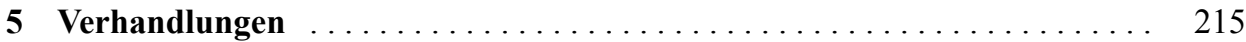

5.1 Veränderungsfaktor: vernetzte und informierte Superkäufer . . . . . . . 215

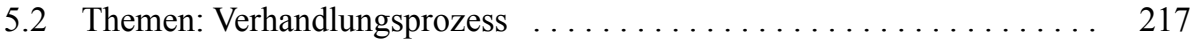

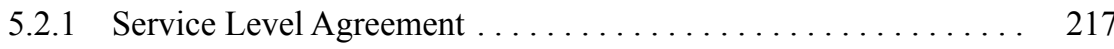

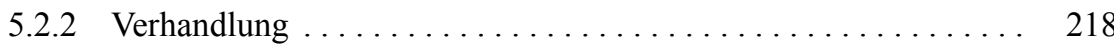

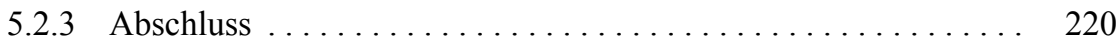

5.2.4 Einwandbehandlung: das doppelte Lottchen . . . . . . . . . . . 222

5.3 Profile: Steher, Primus, Trüffelschwein und Egoist . . . . . . . . . . 225

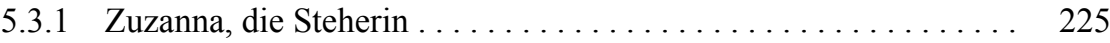

5.3.2 Mikkel, der Primus . . . . . . . . . . . . . . . . . . . . . . . . . . 229

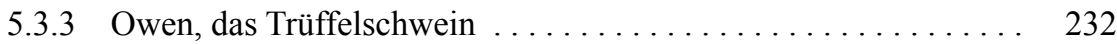

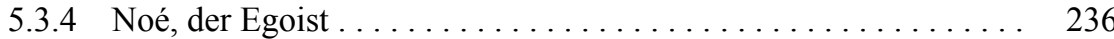

5.4 Coaching-Leitfragen: Verhandlungen und Energie $\ldots \ldots \ldots \ldots \ldots \ldots . \ldots 239$

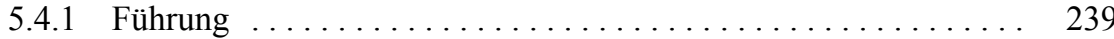

5.4 .2 Mitarbeiter ......................... 240

5.4 .3 Allgemeine, persönliche Fragen . . . . . . . . . . . . . 240

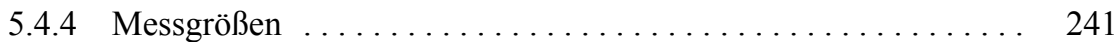

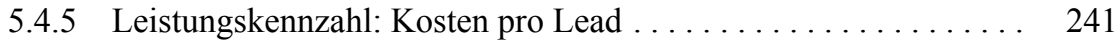

5.5 Leadership: Abschluss und Erfolg . . . . . . . . . . . . . . . . . . . . . 241

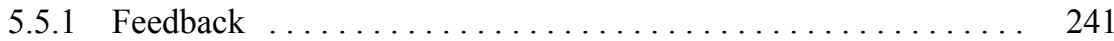

5.5.2 Aus dem Sales-Management-Werkzeugkasten . . . . . . . . . 242

5.6 Anwendung und Ergebnisse: Verhandlungsflexibilität $\ldots \ldots \ldots \ldots \ldots 246$

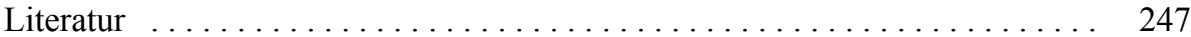

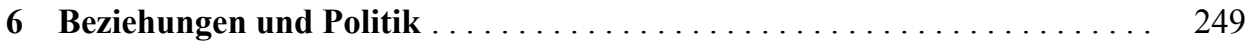

6.1 Veränderungsfaktor: Aufmarsch der Millennials . . . . . . . . . . . . . . . 249

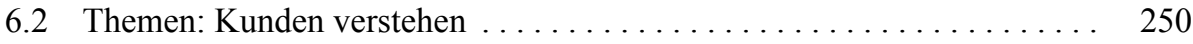

6.2.1 Kundenentwicklung und Accounts . . . . . . . . . . . . . 250

6.2.2 Durchsetzung und Einfühlungsvermögen: Trusted Advisor . . . . 253

6.2 .3 Kundenentscheidungen ................... 256

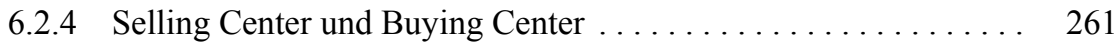

6.2 .5 Kundensicht . . . . . . . . . . . . . . . . . . . . . . . . . . 262

6.2.6 Kundenentwicklungscockpit . . . . . . . . . . . . . . . . 269

6.3 Profile: Berater und Umsetzer . . . . . . . . . . . . . . . . . . . . 273

6.3.1 Marcos, der Ratgeber . . . . . . . . . . . . . . . . . . . . . . . 273

6.3.2 Alicia Christiana, die Füchsin $\ldots \ldots \ldots \ldots \ldots \ldots \ldots \ldots \ldots \ldots$

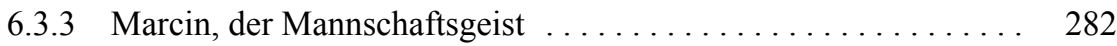

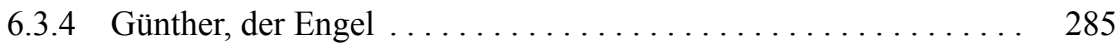

6.4 Coaching-Leitfragen: Vertriebsarbeit in Organisationen . . . . . . . . . . 289

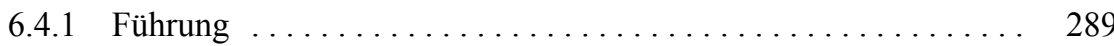

6.4 .2 Mitarbeiter .............................. 289 
6.4.3 Allgemeine, persönliche Fragen . . . . . . . . . . . . . . . . . . . 290

6.4 .4 Messgrößen . . . . . . . . . . . . . . . . . . . . . . 290

6.4.5 Leistungskennzahl: Customer Engagement Level . . . . . . . . 290

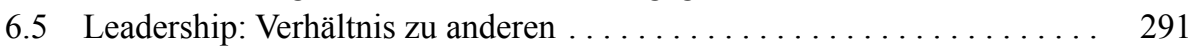

6.5.1 Strategie und Taktik . . . . . . . . . . . . . . . . . . . . 291

6.5.2 Aus dem Sales-Management-Werkzeugkasten . . . . . . . . . . 292

6.6 Anwendung und Ergebnisse: Planen mit Kunden . . . . . . . . . . . . . 299

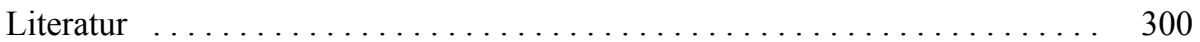

7 Verwandlung zur vertrieblichen Meisterschaft $\ldots \ldots \ldots \ldots \ldots \ldots \ldots \ldots$

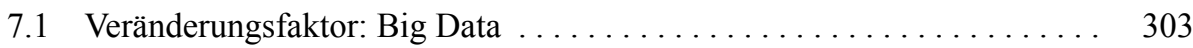

7.2 Themen: Führen und Betreuen . . . . . . . . . . . . . . . . . . . 304

7.2.1 Vom ehrbaren Kaufmann zum Vertriebsmanager . . . . . . . . . 304

7.2 .2 Management und Leadership . . . . . . . . . . . . . . . . 306

7.2.3 Situationsgerechte Führung im Vertrieb . . . . . . . . . . . . . . 308

7.2.4 Definition und Einbettung des Vertriebscoachings $\ldots \ldots \ldots \ldots 311$

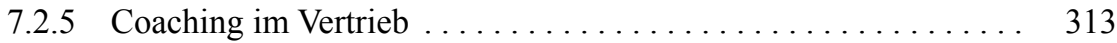

7.2.6 Vertriebscoaching als Philosophie .............. 317

7.2.7 Modell des Vertriebscoachings . . . . . . . . . . . . . . . . . . . . . . 319

7.2.8 Kulturraum als Rahmenbedingung $\ldots \ldots \ldots \ldots \ldots \ldots \ldots \ldots \ldots \ldots$

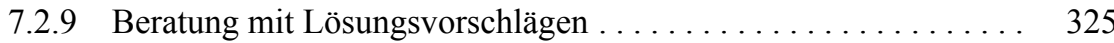

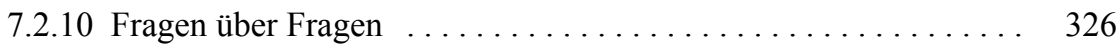

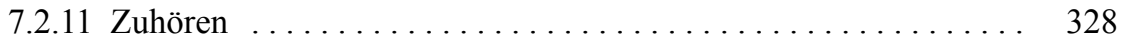

7.2 .12 Vertriebsprofile ....................... 328

7.2.13 Kommunikative Tretminen . . . . . . . . . . . . . . 330

7.3 Profile: Maschinist, Bischof, Terminator und Dekan . . . . . . . . . . 331

7.3.1 Der Maschinist Johannes Mannsheim . . . . . . . . . . . . 331

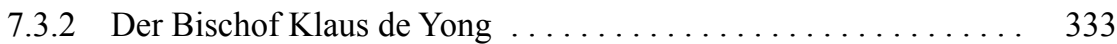

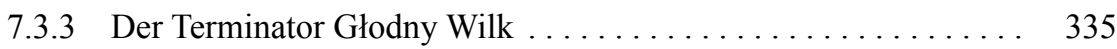

7.3.4 Der Dekan Robert Ganges . . . . . . . . . . . . . . . . . . . . 338

7.4 Coaching-Leitfragen: Betreuung der Vertriebsarbeit . . . . . . . . . . 341

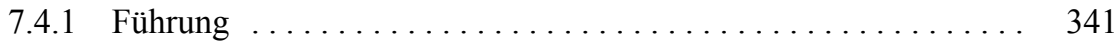

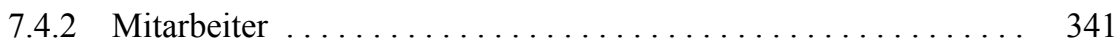

7.4 .3 Messgrößen .......................... 341

7.4.4 Leistungskennzahl: Operating Expense Ratio . . . . . . . . . . . 342

7.5 Leadership: Vertriebsführungskraft als Coach . . . . . . . . . . . . . . . 342

7.5.1 Perspektive Coaching . . . . . . . . . . . . . . . . . . . 342

7.5.2 Aus dem Sales-Management-Werkzeugkasten . . . . . . . . . 343

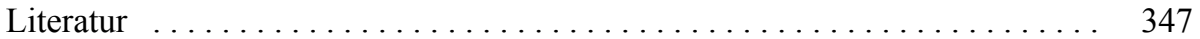


Epilog ... . . . . . . . . . . . . . . . . . . . . . . . . . . . . . 349

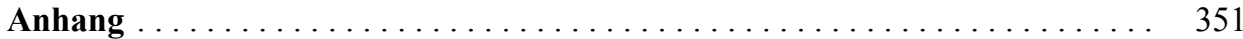

Weiterführende Literatur . . . . . . . . . . . . . . . . . . . . . . . . . . . 367

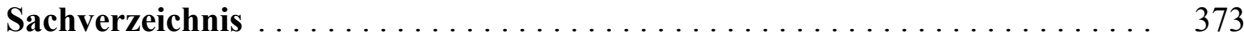




\section{Der Autor}

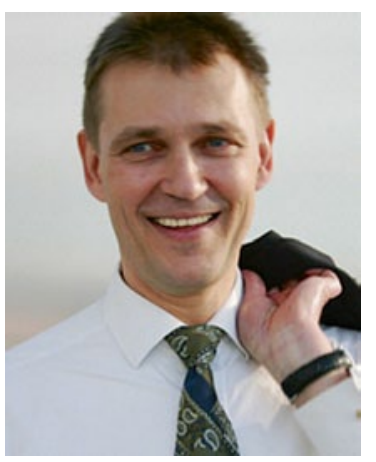

Dr. Nikolaus A. Rauch Jahrgang 1955, gründete 2002 die Business Integrations Services GmbH. Seine Vision ist das optimale Zusammenwirken von Vertrieb, Service und Management. Seit Anfang der 90er Jahre hat er aus unterschiedlichen Perspektiven Vertrieb erlebt und vertrieblich gehandelt: als Verkäufer im Mittelstand, als Account Manager, als Einkäufer von Dienstleistung und Beratung, als Vertriebsleiter, als Geschäftsführer, Berater und aktuell als Partner in der Geschäftsführung des IT-Systemhauses [s.i.g.] in Neu-Ulm. Er verfügt über langjährige Erfahrung im organisatorischen Wandel. So hat er mehrere Jahre in Managementpositionen bei Siemens und Siemens IT Services - dort unter anderem in der Geschäftsführung Deutschland - die weltweite Zusammenführung von Vertrieb und Service verantwortet, den Aufbau einer Service Academy als Vorbild für den Learning Campus des Siemenskonzerns gestaltet und die Integration der Serviceorganisation in die Siemens Business Services GmbH vorbereitet. Er hat in seinem großen Netzwerk bei diversen Firmen in interimistischer Führungsfunktion oder als Berater gearbeitet.

Seine Experten-Schwerpunkte sind Vertriebsmanagement, Vertriebsoptimierungen, Restrukturierungen und Implementierung von Coaching in Vertriebsorganisationen. $\mathrm{Zu}$ seinen besonderen Interessenschwerpunkten zählen analoge Analyseverfahren zu Stärken und Schwächen von Vertriebsorganisationen und Integrationsmanagement.

Nikolaus Rauch lebt mit seiner Frau in München-Fürstenried und hat drei erwachsene Kinder. Er spielt verschiedene Musikinstrumente, wandert gern und besucht weltweit Bildergalerien. Sein Motto: Ganzheitliche Fitness ist das Ergebnis von Disziplin und Respekt vor dem Körper wie dem Geist. Seine Mitarbeiter und Coachees erfahren, dass dies und darüber hinaus die Pflege des persönlichen Netzwerks die entscheidenden Kriterien für langfristigen Vertriebserfolg sind. 